\title{
PROPUESTA Y VALIDACIÓN DE LA HERRAMIENTA DE GESTIÓN DE RIESGOS DEL TRABAJO: GERITRA
}

\author{
Guzmán Franz ${ }^{1}$, Gavilanes Edgar ${ }^{2}$, Jara Oswaldo ${ }^{3}$, Dávila Pablo ${ }^{4}$, Guzman Paula ${ }^{5}$, Cano Víctor ${ }^{6}$. \\ franz.guzman'@uisek.edu.ec., rolando_gavilanes²@hotmail.com., oswaldo.jara ${ }^{3} @$ uisek.edu.ec., pablo.davila@ \\ uisek.edu.ec.,naho.gb $@$ gmail.com., canovictorusfq $@$ hotmail.com. \\ ORCID ${ }^{1}$ : 0000-0002-2018-4009, ORCID ${ }^{2}$ : 0000-0001-9170-6364, ORCID ${ }^{3}$ : 0000-0002-5902-7932, \\ ORCID ${ }^{4}$ : 0000-0002-3166-9300, ORCID 5 : 0000-0002-4797-5564, ORCID ${ }^{6}$ : 0000-0002-1180-5950. \\ Universidad Internacional Sek ${ }^{1}, 2,3,4$ \\ Asociación de profesionales de Seguridad y Salud en el Trabajo Ecuador-APSSTEC 56 \\ Quito-Ecuador
}

Recibido (01/06/20), Aceptado (19/06/20)

\begin{abstract}
Resumen: El objetivo de este trabajo fue presentar y validar la metodología GERITRA de gestión de riesgos del trabajo, una herramienta técnica para identificar y evaluar los riesgos en los puestos de trabajo, permitiendo a los profesionales de seguridad y salud laboral plantear controles operativos, así como el seguimiento de estos, el método empleado es de tipo descriptivo con enfoque semicuantitativo, se empleó la técnica Delphi (consulta a expertos), aplicando a 14 especialistas de la prevención internacionales y 29 especialistas nacionales, las respuestas fueron estructuradas en escala Likert, y la fiabilidad del instrumento la dio el alfa de Cronbach con 0,967, los resultados arrojaron el 97,54\% de aceptabilidad, las conclusiones sugieren que la matriz propuesta es una herramienta, apta para la gestión de riesgos laborales, estructurada en cuatro elementos, la identificación del puesto de trabajo, la identificación de peligros y evaluación de riesgos, la jerarquía de los controles y la gestión del riesgo residual
\end{abstract}

Palabras Clave: Puesto de trabajo, identificación de peligros, evaluación de riesgos, jerarquías de control.

\section{PROPOSAL AND VALIDATION OF THE GERITRA WORK RISK MANAGEMENT TOOL}

\begin{abstract}
The objective is to present and validate the GERITRA methodology of occupational risk management, a technical tool to identify and evaluate risks in the workplace, allowing occupational health and safety professionals to establish operational controls, as well as monitoring of these, the method used is descriptive with a semiquantitative approach, the Delphi technique was used (consultation with experts), applying to 14 international prevention specialists and 29 national specialists, the responses were structured on a Likert scale, and the reliability of the instrument was given Cronbach's alpha with 0.967 , the results showed $97.54 \%$ acceptability, the conclusions suggest that the proposed matrix is a tool, suitable for occupational risk management, structured in four elements, the identification of the job, the identification of hazards and comprehensive risk diagnosis, the hierarchy of controls and the management of the residual risk
\end{abstract}

Keywords: Job position, hazard identification, risk assessment, control hierarchies. 


\section{I.INTRODUCCIÓN}

En la actualidad el marco legal ecuatoriano de seguridad y salud del trabajo establece la necesidad de la identificación, evaluación y control de los riesgos laborales, esto obliga a las organizaciones a generar la información necesaria que les permita adoptar acciones preventivas que tengan como objetivo la eliminación, disminución o sustitución de los riesgos en el trabajo, en [1]

Los principios básicos de la gestión preventiva en [2], [3] de riesgos establecen la necesidad de realizar una evaluación inicial de los mismos, para garantizar la seguridad y salud de los trabajadores, para esto es necesario considerar la naturaleza de la actividad, las características de los puestos de trabajo y de los trabajadores que van a desarrollarlos en [3], para cumplir lo mencionado se han desarrollado varias herramientas que permiten identificar, evaluar y controlar los riesgos laborales, en cada país y/o región se han definido distintos métodos para cumplir con este objetivo. En España el Instituto Nacional de Seguridad y Salud en el Trabajo (INSST) ha definido el método binario de evaluación de riesgos laborales en [4], el Instituto Catalán de Seguridad y Salud Laboral (ICSSL) ha desarrollado el manual para la identificación y evaluación de riesgos laborales en [5], en otros países como Colombia se establece que el empleador tiene como obligación realizar la gestión bajo medidas de identificación de peligros, evaluación de riesgos y establecimiento de controles en [6], [7]mediante la guía para la identificación de peligros y la valoración de los riesgos de seguridad y salud ocupacional en [8].

En Ecuador se han generado medidas legislativas y reglamentarias sobre seguridad y salud en el trabajo, planteándose como secuencia operativa la siguiente: identificación de peligros, medición-evaluación-control de los riesgos, vigilancia de la salud y evaluaciones periódicas en [9], todos los centros de trabajo deben establecer medidas tendientes a eliminar, disminuir y controlar los riesgos laborales, para esto es necesario emplear una metodología que garantice el cumplimiento de la secuencia establecida y mantenga el criterio de la prevención, esta herramienta técnica debe ser reconocida a nivel nacional e internacional, el método debe facilitar la identificación, cuantificación y evaluación de los riesgos en forma inicial y periódica, esto con la finalidad gestionar la seguridad y salud laboral en función de la mejora continua, lo cual garantizará la mejora en las condiciones de seguridad y salud de los trabajado- res, que redundará en la mejora de la calidad de vida de la sociedad ecuatoriana en [10], la normativa ecuatoriana a la fecha no establece metodologías propias para realizar la identificación, medición, evaluación y control de riesgos, que, si bien no es necesario, ya que los métodos reconocidos a nivel internacional suplen esta necesidad, sin embargo, se requiere un método con una herramienta especifica que permita la gestión de riesgos del trabajo, la falta de esta genera que los profesionales de la seguridad y salud ocupacional empleen la metodología que a su parecer sea la más apropiada a su necesidad, generando una falta de criterios homogéneos que no permiten generar una base de datos estandarizada, lo cual dificulta el levantamiento de estadísticas que en lo posterior facilite la toma de decisiones a nivel nacional.

Esta investigación tiene como propósito la presentación y validación de un método y su herramienta técnica de aplicación, que identifique, mida, evalúe, jerarquice el control y realice un seguimiento de los riesgos, técnica que se propondrá a la comunidad ecuatoriana, siendo un instrumento que permita desarrollar el trabajo de manera segura y saludable.

\section{II.DESARROLLO}

El proyecto contemplo dos etapas, la primera de concepción metodológica y una segunda de validación de la herramienta creada.

A.Etapa 1 concepción metodológica. Parte de la conformación de un equipo multidisciplinario de profesionales, con especialidad en seguridad y salud laboral que aporten con conocimientos y experiencia en el área de la seguridad y salud en el trabajo, este equipo a través de una investigación documental estructuró el método, obteniendo una guía metodológica y una herramienta de aplicación en excel, este algoritmo cumple con cuatro fases que se detallan.

a.Fase 1 identificación del puesto de trabajo, corresponde a la descripción del puesto de trabajo a evaluar, se requiere de conocimiento específico sobre los procesos que se ejecutan en la organización, de los puestos de trabajo, de las actividades que se desarrollan y cómo estas pueden influir en la generación de riesgos, así como las deficiencias, las limitaciones de actividad y las restricciones de participación de los trabajadores (discapacitados), que se desempeñan en estas funciones, en la Tabla I se expone el listado de las variables consideradas en este proceso. 
Tabla I.- Variables Matriz GERITRA, Identificación puesto trabajo

\begin{tabular}{lc}
\hline \multicolumn{1}{c}{ Etiqueta } & Variable \\
\hline Proceso & IPT1 \\
Puesto de trabajo & IPT2 \\
Personas con discapacidad & IPT3 \\
Porcentaje de discapacidad & IPT4 \\
Ayudas Técnicas del trabajo & IPT5 \\
Observación & IPT6 \\
Actividad Rutinaria & IPT7 \\
Actividad No Rutinaria & IPT8 \\
Maquinas, Equipos, Sustancias y energías empleadas & IPT9 \\
\hline
\end{tabular}

Fuente: Autor - Nota: IPT Identificación del puesto de trabajo

b.Fase 2 identificación de peligros y evaluación de los riesgos, se identifican los peligros y evalúa su probabilidad de materialización, tomando en cuenta cuatro índices, como se muestra en la tabla II, luego la suma- toria de estos y el análisis de sus consecuencias, nos otorga la valoración del riesgo y el nivel de aceptabilidad en [11].

Tabla II.- Índices Guía del usuario Matriz GERITRA, Variables de probabilidad

\begin{tabular}{|c|c|c|c|c|c|}
\hline índice & Valor & $\begin{array}{l}\text { personas } \\
\text { expuestas }\end{array}$ & $\begin{array}{l}\text { procedimientos } \\
\text { existentes }\end{array}$ & $\begin{array}{l}\text { capacitación y } \\
\text { capacidades } \\
\text { humanas }\end{array}$ & $\begin{array}{l}\text { exposición al riesgo } \\
\text { (frecuencia) }\end{array}$ \\
\hline 1 & Baja & $1-5$ & $\begin{array}{l}\text { existen y son } \\
\text { satisfactorios y } \\
\text { suficientes }\end{array}$ & $\begin{array}{l}\text { Personal entrenado, } \\
\text { conoce el peligro y } \\
\text { lo previene }\end{array}$ & $\begin{array}{l}\text { personal expuesto al peligro } \\
\text { en raras veces, de manera } \\
\text { muy inusual o en largos } \\
\text { periodos transcurridos. }\end{array}$ \\
\hline 2 & Media & $6-12$ & $\begin{array}{c}\text { existen } \\
\text { parcialmente o } \\
\text { no son } \\
\text { satisfactorios o } \\
\text { suficientes }\end{array}$ & $\begin{array}{l}\text { personal } \\
\text { parcialmente } \\
\text { entrenado, conoce el } \\
\text { peligro, y no toma } \\
\text { acciones de control. }\end{array}$ & $\begin{array}{l}\text { personal expuesto al peligro } \\
\text { de manera eventual, en } \\
\text { algunas ocasiones, lapsos de } \\
\text { tiempo medio, no seguido }\end{array}$ \\
\hline 3 & Alta & $>12$ & no existen & $\begin{array}{ll}\text { personal no } & \text { no } \\
\text { entrenado, no } & \text { no } \\
\text { conoce el peligro, no } \\
\text { toma acciones de } \\
\text { control }\end{array}$ & $\begin{array}{l}\text { personal expuesto al peligro } \\
\text { de manera continua, } \\
\text { frecuente, siempre o casi } \\
\text { siempre, recurrente, } \\
\text { periodos de tiempo cortos, } \\
\text { seguidos }\end{array}$ \\
\hline
\end{tabular}

Fuente: Autor - Nota: Variables cuya sumatoria ayudan a definir la probabilidad

Para su aplicación se recomienda que el equipo de análisis y evaluación lo haga in situ, observando las diferentes áreas y puestos de trabajo, verificando las actividades junto a cada responsable, evaluando las máqui- nas, equipos, herramientas, factores ambientales, que se encuentren y que a su vez puedan generar algún tipo de riesgo, luego con el apoyo de la Tabla III se evalúa el nivel de severidad 
Tabla III. Guía del usuario MATRIZ GERITRA, índice de consecuencias-severidad

\begin{tabular}{|c|c|c|}
\hline Índice & $\begin{array}{l}\text { calific } \\
\text { ación }\end{array}$ & consecuencias-severidad \\
\hline 1 & $\begin{array}{l}\text { ligera } \\
\text { mente } \\
\text { dañino }\end{array}$ & $\begin{array}{l}\text { (seguridad) sin lesión/ lesión sin } \\
\text { discapacidad, pequeños cortes } \\
\text { magulladuras. } \\
\text { (salud ocupacional) malestar, molestias e } \\
\text { irritación, dolor de cabeza, irritación de } \\
\text { ojos, disconfort. }\end{array}$ \\
\hline 2 & $\begin{array}{c}\text { Dañin } \\
\text { o }\end{array}$ & $\begin{array}{l}\text { (seguridad) lesión con incapacidad } \\
\text { temporal fracturas menores, cortes, } \\
\text { quemaduras, conmociones, torceduras } \\
\text { importantes, } \\
\text { (salud ocupacional) daño a la salud } \\
\text { reversible dermatitis, asmas, trastornos } \\
\text { musculoesqueléticos. }\end{array}$ \\
\hline & & $\begin{array}{llll}\text { (seguridad) } & \text { lesión/lesiones } & \text { múltiples } \\
\text { fatales } & \text { o } & \text { con } & \text { incapacidad }\end{array}$ \\
\hline 3 & $\begin{array}{c}\text { extrem } \\
\text { adame } \\
\text { nte } \\
\text { dañino }\end{array}$ & $\begin{array}{l}\text { permanente/muerte, amputaciones, } \\
\text { fracturas mayores. } \\
\text { (salud ocupacional) daño a la salud } \\
\text { irreversible, intoxicaciones masivas, } \\
\text { pérdida auditiva, cáncer y otras } \\
\text { enfermedades, enfermedades crónicas. }\end{array}$ \\
\hline
\end{tabular}

Fuente: Autor - Nota: tabla orientativa para evaluar el nivel de consecuencias o severidad

Finalmente, en la Tabla IV. se expone el detalle de variables consideradas en la fase 2 correspondiente al algoritmo planteado para identificar los peligros y evaluar de manera general los riesgos, considerando los factores de riesgo del medio ambiente físico, por conta- minantes químicos-bilógicos, ergonómicos, psicosociales, a la seguridad de las personas en donde están considerados mecánicos y locativos y los factores de riesgo ambiental considerando el daño al entorno inmediato a consecuencia de los procesos ejecutados.

Tabla IV.- Variables Matriz GERITRA, Identificación peligros y evaluación de riesgos

\begin{tabular}{lc}
\hline Etiqueta & Variable \\
\hline Peligro & IPER1 \\
Cantidad de personas expuestas & IPER2 \\
Índice de personas expuestas & IPER3 \\
Índice de procedimientos existentes & IPER4 \\
Índice de capacitaciones y capacidades humanas & IPER5 \\
Índice de exposición al riesgo & IPER6 \\
Probabilidad & IPER7 \\
Consecuencias (severidad) & IPER8 \\
Valoración del riesgo & IPER9 \\
Nivel de riesgo & IPER10 \\
Clasificación de los riesgos & IPER11 \\
\hline
\end{tabular}

Fuente: Autor - Nota: IPER. Identificación de peligros y evaluación de riesgos

c.Fase 3 jerarquía de los controles de la seguridad, de manera jerarquizada para eliminar, sustituir, reducir correspondiente a la aplicación de controles operativos o controlar los riesgos detectados, en base a recomen- 
daciones establecidas en la Norma ISO 45001:2018 en [12], desglosándolos para que sean más entendibles y sustituyendo la categoría equipos de protección personal por control sobre él trabajador.

Tabla V.- Variables Matriz GERITRA, Jerarquía de los controles de seguridad

\begin{tabular}{lc}
\hline \multicolumn{1}{c}{ Etiqueta } & Variable \\
\hline Eliminar & JCS1 \\
Sustituir & JCS2 \\
Control de ingeniería y diseño & JCS3 \\
Control administrativo & - \\
Normas, planes procedimientos y permisos & JCS4 \\
Horarios, descansos y rotación de puestos & JCS5 \\
Señalización & JCS6 \\
Control sobre él trabajador & - \\
Formación, información y capacitación & JCS7 \\
Protección colectiva e individual & JCS8 \\
\end{tabular}

Fuente: Autor - Nota: JCS. Jerarquía de los controles de seguridad

d.Fase 4 gestión del riesgo residual, se refiere a la actuación o tratamiento que debe darse a los riesgos, luego de la ejecución de los controles operativos propuestos en [13], esto con el fin de generar un proceso de mejora continua, estableciendo acciones de monitoreo y vigilancia, el proceso inicia con una reevaluación aplicando los mismos pasos descritos en la fase 2 de identificación de peligros y evaluación de riesgos, luego con la intervención médica ocupacional se describen los efectos sobre la salud de los trabajadores y los protocolos para vigilar de manera permanente su salud, finalmente en esta misma fase se propone una fecha para controlar el cumplimiento de los objetivos establecidos y se detallan las acciones de mejora continua, siendo el momento de proponer las metodologías específicas para evaluar los riesgos que se han detectado y que requieren de un tratamiento más específico, en la Tabla VI. Se expone el listado de variables.

Tabla VI.- Variables Matriz GERITRA, Gestión de riesgo residual

\begin{tabular}{|c|c|}
\hline Etiqueta & Variable \\
\hline Valoración del riesgo & GRR1 \\
\hline Efectos sobre la salud de los trabajadores & GRR2 \\
\hline Vigilancia de la salud & GRR3 \\
\hline Fecha de control de inspecciones & $\begin{array}{l}\text { GRR4 } \\
\text { GRR5 }\end{array}$ \\
\hline
\end{tabular}

Actividades de mejora continua

Fuente: Autor Nota: GRR. Gestión de riesgo residual

A.Etapa 2 validación de la herramienta. Luego del proceso de construcción de la metodología correspondió validar el trabajo sometiéndolo al juicio de expertos, considerando que es un tema netamente técnico que debe tratarse con especialistas que dominen el arte, planteándose que estos sean tanto del ámbito local como internacional. 


\section{III.METODLOGÍA}

B.Diseño del cuestionario, damos inicio partir de la construcción del cuestionario en [14] con 33 variables determinadas en las fases del método GERITRA, cuyo objetivo principal es evaluar el riesgo laboral de manera general que presenta una organización, se compilan y se plantea en escala Likert en [15], misma que define 6 niveles que son de tipo ordinal, los niveles y su codificación se expone en la Tabla VII.

\section{Tabla VII.- Criterio de valoración. Matriz GERITRA}

\begin{tabular}{lc}
\hline Etiquetas de valores & Codificación \\
\hline Totalmente inaplicable & 1 \\
Inaplicable & 2 \\
Poco aplicable & 3 \\
Aplicable & 4 \\
Muy aplicable & 5 \\
Totalmente aplicable & 6 \\
\hline
\end{tabular}

Fuente: Elaboración, análisis e interpretación de encuestas, Alamios

C.Consistencia interna del instrumento, una vez desarrollado el cuestionario y con el fin de verificar su firmeza en la estructura, se aplica el coeficiente de fiabilidad alfa de Cronbach en [16], [17] lo cual nos permite medir el constructo correlacionado mediante las seis opciones de respuesta (Tabla 7), el valor obtenido de la aplicación fue de 0,967 , en valor muy aproximado a una unidad, lo cual nos indica que mientras más se acerque a 1, mayor es la consistencia interna de las variables analizadas. Esto nos permite inferir que el cuestionario es adecuado, pues cumple con los requisitos de confiablidad y validez en [18]. Determinando así que las respuestas de cada una de las 33 variables del cuestionario son lo suficientemente coherentes entre sí.

D.Selección de la metodología de investigación. Para la validación del Método GERITRA, se aplicó el método Delphi en [19] (consulta a expertos nacionales e internacionales en Seguridad y Salud Laboral), en el periodo de agosto a noviembre del 2019 , la consulta a expertos, entre otros métodos científicos cualitativos, es el más utilizado a nivel mundial, destacando su utilidad en la investigación de las ciencias sociales en [20]. Esta es una técnica de obtención de información mediante la consulta a expertos, ya que debido a su experiencia profesional o formación académica tienen la capacidad de emitir juicios de valor respaldados por su experiencia y experticia.

.Selección de especialistas. Se realiza la selección de 43 expertos considerando el conocimiento, experiencia y prestigio profesional en sus áreas de desempeño, la elección de los expertos es un proceso fundamental en el método Delphi, y de esto dependerá la confiabilidad de los resultados a obtener en [21]. Las características propuestas son:

-Experiencia en seguridad y salud/gerentes de empresas consultoras de seguridad y salud en el trabajo,

-Especialistas en seguridad y salud en el trabajo que apliquen y prueben la metodología,

-Expertos en temas relacionados con seguridad y salud en el trabajo con grado de doctorado y maestría,

-Amplia experiencia/representante de gremios profesionales seguridad y salud en el trabajo,

-Médicos Ocupacionales,

-Expertos de categoría nacional e internacional.

A continuación, se expone en la Tabla VIII, conformación del grupo de especialistas consultados a quienes luego de explicar los objetivos de la encuesta, se les distribuyo el documento. 
Tabla VIII.- Grupo de expertos, Método GERITRA

\begin{tabular}{lccc}
\hline \multicolumn{1}{c}{ Cargo } & $\begin{array}{c}\text { \# expertos } \\
\text { nacionales }\end{array}$ & $\begin{array}{c}\text { \# expertos } \\
\text { internacionales }\end{array}$ & Total \\
\hline Gerente de empresa Consultora de SST & 7 & 4 & 11 \\
Especialista técnico en SST & 16 & 3 & 16 \\
Docente universitario en SST & 3 & 4 & 6 \\
Docente universitario, director de departamento de SST & 2 & 2 & 6 \\
Representante de gremios profesionales en SST & 1 & 1 & 2 \\
Medico ocupacional & 29 & 14 & 2 \\
Total & & & 43 \\
\hline
\end{tabular}

Fuente: Autor - Nota: SST. Seguridad y salud en el trabajo

E.Distribución del cuestionario. La invitación a participar de la validación a los expertos fue enviada vía electrónica, mediante un documento que describió el proceso de construcción del método [22] (antecedentes, objetivos, pasos del método, la herramienta del método en Excel - Matriz GERITRA y el cuestionario para medir el nivel de aplicabilidad de la matriz), sometido a juicio de los especialistas, el cuestionario incluyo, luego de cada sección de preguntas de las cuatro fases del método, un espacio para observaciones y recomendaciones, el objetivo fue valorar el contenido de las variables del Método GERITRA.

F.Análisis de resultados. Al obtener los resultados se realizó la organización, análisis y retroalimentación de los datos de acuerdo con las observaciones emitidas por el grupo de expertos, los datos obtenidos fueron analizados estadísticamente mediante el programa estadístico IBM SPSS software V22 en [23] los resultados representan la puntuación que dieron los expertos a cada una de las variables, de modo que si el valor obtenido es o se acerca a 6 representa que las variables evaluadas tienen alta aplicabilidad a criterio del grupo experto, caso contrario ocurre, si la puntuación que dieron los expertos es igual o se acerca a 1. Las respuestas obte- nidas determinaron un nivel de aceptación del 97,54\%, con tres recomendaciones cualitativas a la forma de presentación de la tabla (formato Excel), más no a la estructura del método planteado, existiendo un grado de convergencia de las estimaciones individuales superior al $80 \%$ en [22], lo cual permitió determinar la viabilidad de las variables, y por tanto, la validez del método propuesto, bajo esta consideración no fue necesario aplicar una segunda roda del cuestionario como sugiere la metodología Delphi, los resultados se presentan en el siguiente apartado.

\section{IV.RESULTADOS}

Para la primera fase (identificación del puesto de trabajo), se obtuvo el $97.93 \%$ sumando a quienes calificaron al menos como aplicable (desde 4 a 6 en la escala de Likert), el $2.07 \%$ indicó que es poco aplicable (3 en la escala de Likert). Las variables que obtuvieron una valoración desfavorable (valores entre 1 y 3 ) por parte de los expertos están relacionadas con: la identificación de discapacidades, porcentaje de discapacidad y actividades no rutinarias. En la Tabla IX, se muestra las frecuencias de los resultados para cada variable expuesta en la (fase 1).

Tabla IX.- Resultados de frecuencias relativas Método GERITRA, Identificación del puesto de trabajo

\begin{tabular}{ccccc}
\hline Variable & 6 en $(\%)$ & 5 en $(\%)$ & 4 en $(\%)$ & 3 en $(\%)$ \\
\hline IPT1 & 65,12 & 18,60 & 13,95 & 2,33 \\
IPT2 & 69,77 & 16,28 & 13,95 & 0,00 \\
IPT3 & 60,47 & 20,93 & 18,60 & 0,00 \\
IPT4 & 53,49 & 25,58 & 18,60 & 2,33 \\
IPT5 & 55,81 & 23,26 & 18,60 & 2,33 \\
IPT6 & 53,49 & 23,26 & 13,95 & 9,30 \\
IPT7 & 69,77 & 16,28 & 13,95 & 0,00 \\
IPT8 & 65,12 & 16,28 & 16,28 & 2,33 \\
IPT9 & 58,14 & 20,93 & 20,93 & 0,00 \\
\% Total & 61,24 & 20,16 & 16,54 & 2,07 \\
\hline
\end{tabular}

Fuente: Autor - Nota: los valores de 1 y 2 no se presentaron en esta etapa 
En las preguntas de la segunda fase (identificación de peligros y evaluación de riesgos), se obtuvo el 96.83\% sumando a quienes calificaron al menos como aplicable (desde 4 a 6 en la escala de Likert), el 3.17\% indicó que es poco aplicable ( 3 en la escala de Likert). En la Tabla X, se muestra las frecuencias de los resultados para cada variable de la (fase 2). El resultado global de la aplicabilidad del Método GERITRA, se obtuvo que

Tabla X.- Resultados de frecuencias relativas Método GERITRA, Identificación peligros y evaluación de riesgos

\begin{tabular}{ccccc}
\hline Variable & 6 en $(\%)$ & 5 en $(\%)$ & 4 en $(\%)$ & 3 en $(\%)$ \\
\hline IPER1 & 83,72 & 4,65 & 11,63 & 0,00 \\
IPER2 & 79,07 & 11,63 & 9,30 & 0,00 \\
IPER3 & 62,79 & 23,26 & 6,98 & 6,98 \\
IPER4 & 55,81 & 18,60 & 18,60 & 6,98 \\
IPER5 & 60,47 & 13,95 & 20,93 & 4,65 \\
IPER6 & 67,44 & 13,95 & 13,95 & 4,65 \\
IPER7 & 58,14 & 20,93 & 13,95 & 6,98 \\
IPER8 & 67,44 & 18,60 & 13,95 & 0,00 \\
IPER9 & 67,44 & 18,60 & 11,63 & 2,33 \\
IPER10 & 62,79 & 20,93 & 13,95 & 2,33 \\
IPER11 & 67,44 & 16,28 & 16,28 & 0,00 \\
\% Total & 66,60 & 16,49 & 13,74 & 3,17 \\
\hline
\end{tabular}

Fuente: Autor - Nota: los valores de 1 y 2 no se presentaron en esta etapa

En la batería de preguntas de la tercera fase (jerarquía de los controles de seguridad y salud), se obtuvo el $99.13 \%$ sumando a quienes calificaron al menos como aplicable (desde 4 a 6 en la escala de Likert), el $0.87 \%$ indicó que es poco aplicable ( 3 en la escala de Likert). En la Tabla XI, se muestra las frecuencias de los resultados para cada variable (fase 3).

Tabla XI.- Resultados de frecuencias relativas Método GERITRA, Jerarquía de los controles de seguridad

\begin{tabular}{ccccc}
\hline Variable & 6 en $(\%)$ & 5 en $(\%)$ & 4 en $(\%)$ & 3 en $(\%)$ \\
\hline JCS1 & 65,12 & 16,28 & 13,95 & 4,65 \\
JCS2 & 69,77 & 13,95 & 16,28 & 0,00 \\
JCS3 & 74,42 & 16,28 & 9,30 & 0,00 \\
JCS4 & 76,74 & 13,95 & 9,30 & 0,00 \\
JCS5 & 72,09 & 18,60 & 9,30 & 0,00 \\
JCS6 & 69,77 & 18,60 & 11,63 & 0,00 \\
JCS7 & 72,09 & 13,95 & 13,95 & 0,00 \\
JCS8 & 69,77 & 13,95 & 13,95 & 2,33 \\
\% Total & 71,22 & 15,70 & 12,21 & 0,87 \\
\hline
\end{tabular}

Fuente: Autor - Nota: los valores de 1 y 2 no se presentaron en esta etapa

En las preguntas aplicadas a cuarta y última fase (gestión del riesgo residual) se obtuvo el 96.28\%, sumando a quienes calificaron al menos como aplicable (desde 4 a 6 en la escala de Likert), 3.26\% sumando a quienes calificaron al menos como poco aplicable (3 en la escala de Likert) y el $0.87 \%$ sumando a quienes calificaron al menos como inaplicable (1 en la escala de Likert). En la Tabla XII, se muestra las frecuencias de los resultados para cada variable (fase 4). 
Tabla XII.- Resultados de frecuencias Método GERITRA, Gestión de riesgo residual

\begin{tabular}{cccccc}
\hline Variable & 6 en $(\%)$ & 5 en $(\%)$ & 4 en $(\%)$ & 3 en $(\%)$ & 1 en $(\%)$ \\
\hline GRR1 & 65,12 & 20,93 & 11,63 & 2,33 & 0,00 \\
GRR2 & 67,44 & 20,93 & 9,30 & 2,33 & 0,00 \\
GRR3 & 72,09 & 11,63 & 11,63 & 4,65 & 0,00 \\
GRR4 & 74,42 & 9,30 & 11,63 & 4,65 & 0,00 \\
GRR5 & 72,09 & 16,28 & 6,98 & 2,33 & 2,33 \\
\% Total & 70,23 & 15,81 & 10,23 & 3,26 & 0,47 \\
\hline
\end{tabular}

Fuente: Autor - Nota: Para el valor de 2 no se presentaron respuestas en esta etapa

El resultado global de la aplicabilidad del Método GERITRA, se obtuvo que el 97,54\%, sumando a quienes calificaron como muy aplicable y aplicable (desde 4 a 6 en la escala de Likert), el 2,46\% sumando a quienes calificaron como inaplicable a totalmente inaplicable (de 1 a 3 en escala Likert).

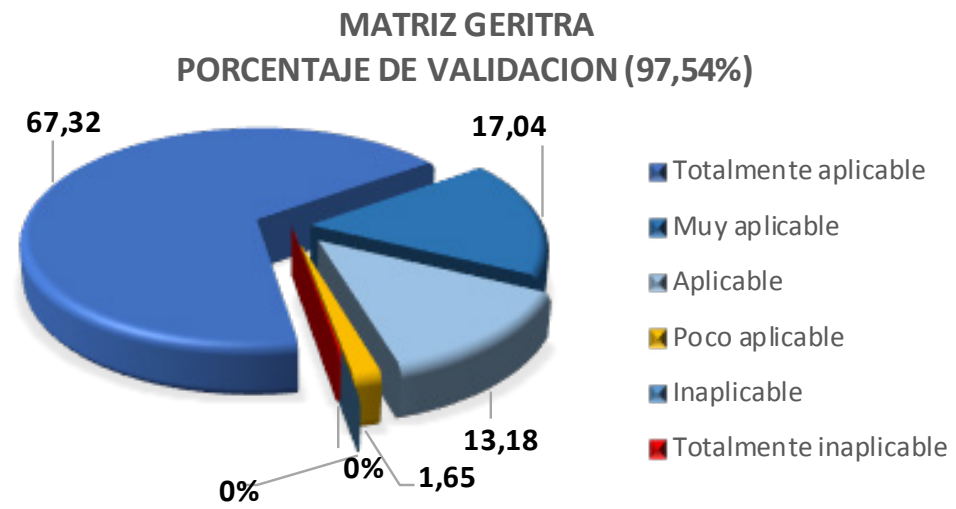

Figura 1. Resultado global de validación- Matriz GERITRA

\section{V.CONCLUSIONES}

Un equipo de profesionales de la prevención de riesgos laborales, con el respaldo de la academia y de la asociación de profesionales de seguridad y salud en el trabajo del Ecuador presentó una metodología para identificación evaluación control, seguimiento y vigilancia de los riesgos presentes en los espacios laborales que comprende 4 fases claramente definidas

El instrumento de análisis (cuestionario de preguntas) aplicado a los especialistas, fue adecuadamente validado a través del coeficiente Alfa de Cronbach con un valor de $(0,967)$, lo cual le otorgó confianza y soporte de validez.

Con los datos obtenidos del cuestionario entregado a 43 especialistas se obtuvo un resultado del 97,54\% alcanzando un alto nivel de aceptabilidad, lo cual permitió no realizar una segunda ronda de preguntas como recomienda la metodología Delphi.

Bajo los parámetros analizados a lo largo del presente artículo, en base a los datos obtenidos de la encuesta aplicada a los especialistas de seguridad y salud en el trabajo tanto del ámbito nacional como internacional, el método GERITRA es totalmente válido e incluye:

-Identificación del puesto de trabajo

- Identificación de peligros y evaluación de riesgos

-Jerarquía de los controles en la seguridad y salud en el trabajo

-Gestión de riesgo residual

La estandarización en el uso de un método para desarrollar una gestión de seguridad y salud del trabajo, permitirá que los resultados totales y/o parciales que se generen sean útiles para la conformación de bases de datos, y que estas sean comparables entre sí, esto permitirá levantar estadísticas que revelen el estado de la gestión en SST a nivel nacional o regional, información con la cual los entes de control podrán tomar decisiones fundamentadas, que apoyen la prevención y control de 
los riesgos laborales.

\section{REFERENCIAS}

[1]E. Ecuatoriano, «Portal único de trámites ciudadanos,» 1711 1986. [En línea]. Available: https://www. gob.ec/sites/default/files/regulations/2018-11/Documento_Reglamento-Interno-Seguridad-Ocupacional-Decreto-Ejecutivo-2393_0.pdf. [Último acceso: 12 febrero 2020].

[2]A. Gómez García , F. Algora Buenafé y P. Suasnavas Bermudez, «Notificación de Accidentes de Trabajo y Posibles Enfermedades Profesionales en Ecuador, 2010-2015,» Scielo, vol. 18, n 57, pp. 166-172, 2016.

[3]E. Español, «Boletin Oficial del Estado,» 8 noviembre 1995. [En línea]. Available: https://www.boe.es/ buscar/pdf/1995/BOE-A-1995-24292-consolidado.pdf. [Último acceso: 15 febrero 2020].

[4]INSST, «Instituto Nacional de Seguridad y Salud en el Trabajo,» 105 2000. [En línea]. Available: https:// www.insst.es/documents/94886/96076/Evaluacion riesgos.pdf/1371c8cb-7321-48c0-880b-611f6f380c1d. [Último acceso: 14 febrero 2020].

[5]C. Genralitat, «Manual para la identificación y evaluación de riesgos laborales. _Versión 3.1,» 8 diciembre 2006. [En línea]. Available: https://www.gencat.cat/ treball/doc/doc_21212475_2.pdf. [Último acceso: 14 febrero 2020].

[6]M. Trabajo, «Ministerio del Trabajo,» 26 mayo 2015. [En línea]. Available: https://www.mintrabajo.gov.co/ documents/20147/0/DUR+Sector+Trabajo+Actualizado $+a+15+$ de + abril ++ de +2016 .pdf/a32b1dcf-7a4e8a37-ac16-c121928719c8. [Último acceso: 14 febrero 2020].

[7]E. García Machin y A. Granda, «La inspección de la seguridad y salud en el trabajo en el nuevo contexto de las relaciones de trabajo,» Salud de los Trabajadores, vol. 20, nº 2, pp. 205-213, 12 diciembre 2012.

[8]ICONTEC, «Instituto Colombiano de Normas Técnicas y Certificación,» 01 agosto 2010. [En línea]. Available: https://idrd.gov.co/sitio/idrd/sites/default/files/ imagenes/gtc450.pdf. [Último acceso: 1402 2020].

[9]IESS, «Portal unico de tramites ciudadanos,» 12 julio 2016. [En línea]. Available: https://www.gob.ec/sites/default/files/regulations/2018-10/C.D.\%20513.pdf. [Último acceso: 14 febrero 2020].

[10]CAN, «Portal de trámites ciudadanos,» 07 mayo 2004. [En línea]. Available: https://www.gob.ec/sites/ default/files/regulations/2018-11/Documento_Decisi\%C3\%B3n-Acuerdo-Cartagena-584.pdf. [Último acceso: 14 febrero 2020].

[11]J. A. Chopitea Cantos y L. J. Delgado Arteaga, «Repositorio Dspace,» 2608 2014. [En línea]. Available:
http://repositorio.unp.edu.pe/handle/UNP/415.

[12]J. Obando Montenegro, M. Sotolongo Sanchez y E. Villa Gonzalez del Pino, «Evaluación del desempeño de seguridad y salud en una empresa de impresión,» Ingeniería Industrial, vol. 40, $\mathrm{n}^{\circ} 2$, pp. 136-147, 1 agosto 2019.

[13]A. Andrés Álvarez y L. Gómez Fernández, Guía de aplicación de la Norma UNE-ISO/IEC 27001 sobre Sguridad en Sistemas de Información para Pymes 2da edición, Madrid: AENOR, 2012.

[14]A. Escofet, P. Folgueiras, E. Luna y B. Palou , «Elaboración y validación de un cuestionario para la valoración de proyectos de aprendizaje-servicio,» Revista Mexicana de Investigación Educativa, vol. 21, n ${ }^{\mathrm{o}} 70$, pp. 929-949, 2016.

[15]A. Alamios Chica y J. Castejón Costa , «Elaboración, análisis e interpretación de encuestas, cuestionarios y escalas de opinión,» Universidad de Alicante, 4 junio 2006. [En línea]. Available: https://rua.ua.es/dspace/bitstream/10045/20331/1/Elaboraci\%c3\%b3n,\%20 an $\%$ c3\%a1lisis\%20e\%20interpretaci\%c3\%b3n.pdf. [Último acceso: 14 febrero 2020].

[16]D. Frías Navarro, «Apuntes de consistencia interna de las puntuaciones de un instrumento de medida,» Uniersidad de Valencia, 01 Noviembre 2019. [En línea]. Available: https://www.uv.es/ friasnav/AlfaCronbach. pdf. [Último acceso: 14 febrero 2020].

[17]P. M. vallejo, Estadística Aplicada a las Ciencias Sociales, . Madrid: UNIVERSIDAD PONTIFICIA COMILLAS, 2008.

[18]Y. González-Ortega, «Instrumento Cuidado y comportamiento profesional: validez y confiabilidad,» Aquichan, vol. 8, nº 2, pp. 170-182, 2008.

[19]M. García Valdéz y M. Suarez Marín , «Empleo del método Delphi en investigaciones sobre salud publicadas en revistas científicas cubanas,» Revista Cubana de INformación en Ciencias de la Salud, vol. 24, nº 2, pp. 133-144, 2013.

[20]M. E. García Rúiz y F. Lena Acebo, «Aplicación del metodo delphi en el diseño de una investigación cuantitativa sobre el fenómeno FABLAB,» Empiria, Revista de Metodología de Cincias Sociales, vol. 40, $\mathrm{n}^{\circ}$ 1, pp. 129-166, 16 mayo 2018.

[21]G. Aponte Figueroa, M. A. Cardozo Montilla y M. Melo Rosa , «Método DELPHI: aplicaciones y posibilidades en la gestión prospectiva de la investigación y desarrollo,» Revista Venezolana de Análsis de Coyuntura, vol. XVIII, no 1, pp. 41-52, 2012.

[22]E. López Gómez, «EL Método Delphi en la Investigación Actual en Educación: Una Revisión Teórica y Metodológica,» Edcuación XX1, vol. 21, nº 1, pp. 1740, 2018. 
[23]E. Bausela Herreras, «SPSS un INTRUMENTO DE ANÁLISIS DE DATOS CUNATITATIVOS,» Re-

\section{RESUMEN CURRICULAR}

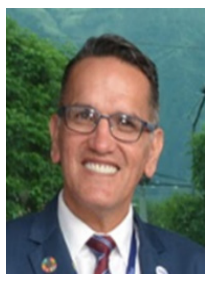

MGs. FranzPaul Guzmán Galarza. Catedrático investigador, Magister en Seguridad, Salud y Ambiente por la Universidad Central del Ecuador, experto en prevención de riesgos laborales por Universidad de Hueva de España, Lcdo. en Ciencias de la Educación, Lcdo. en Ciencias Militares, presidente de la Asociación de Profesionales de Seguridad y Salud en el Trabajo del Ecuador, Auditor líder ERCA ISO 45001:2018

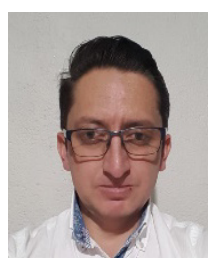

Ing. Edgar Rolando Gavilanes Arellano. Ingeniero en Seguridad y Salud Ocupacional de la Universidad Internacional SEK, capacitador en Seguridad y Salud en el Trabajo en Corposuper

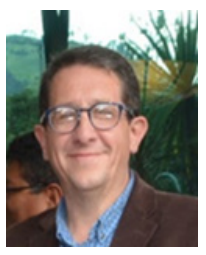

PhD. Jorge Oswaldo Jara Díaz, En filosofía y economía de la salud, Magister en Seguridad, Salud y Ambiente por la Universidad Central del Ecuador y la Universidad de Hueva de España, Máster en Ergonomía, Doctor en medicina y cirugía general, Catedrático de la Ingeniería en Seguridad y Salud Ocupacional de Universidad Internacional SEK, Consultor independiente Auditor líder ISO 45001:2018 vista de Informática Educativa y Medios Audiovisuales, vol. 2, no 4, pp. 62-69, 2005.

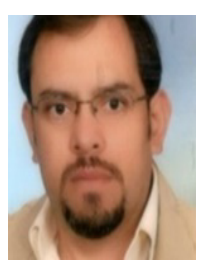

MSc. Pablo Ramiro Dávila, Docente investigador de la Facultad de Ciencias del trabajo y Comportamiento Humano, Carrera de Ingeniería en Seguridad y Salud Ocupacional de la Universidad SEK, Magister en gestión y educación superior, Magister en ciencias Ambientales, Ingeniero Geógrafo.

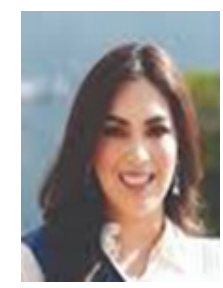

Ing. Paula Nahomy Guzmán Basurto, Ingeniera en Seguridad y Salud Ocupacional, Jefe de Seguridad y Salud en Ecuaferrallas \& Encofrados, empresa del Consorcio Línea 1 del Metro de Quito, maestrante, seguridad en la construcción de edificaciones, Universidad de Sevilla España

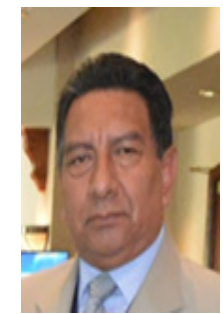

MGs. Víctor Hugo Cano Apolo. Magister en Seguridad, Salud y Ambiente por la Universidad San Francisco de Quito y la Universidad de Hueva de España, Licenciado en Ciencias Militares, Doctor en Ciencias de la Educación, Examinador de competencias Laborales en la empresa pública Espe Innovativa EP y Consultor independiente. Auditor líder ERCA ISO 45001:2018 